PROCEEDINGS OF THE

AMERICAN MATHEMATICAL SOCIETY

Volume 137, Number 2, February 2009, Pages 531-540

S 0002-9939(08)09711-6

Article electronically published on October 6, 2008

\title{
GRADIENTS OF LAPLACIAN EIGENFUNCTIONS ON THE SIERPINSKI GASKET
}

\author{
JESSICA L. DEGRADO, LUKE G. ROGERS, AND ROBERT S. STRICHARTZ
}

(Communicated by Michael T. Lacey)

\begin{abstract}
We use spectral decimation to provide formulae for computing the harmonic tangents and gradients of Laplacian eigenfunctions on the Sierpinski Gasket. These formulae are given in terms of special functions that are defined as infinite products.
\end{abstract}

\section{INTRODUCTION}

There are few functions more ubiquitous in Euclidean analysis than the sine, cosine and exponential, which are the eigenfunctions of the Laplacian on an interval in $\mathbb{R}$. In the theory of analysis on fractals, the Laplacian eigenfunctions arguably have an even more prominent role, as the Laplacian is the fundamental differential operator on which the analysis is based. Despite this, there are a number of interesting open questions about the structure of such eigenfunctions. In this paper we consider the local behavior of Laplacian eigenfunctions on the Sierpinski Gasket (SG), in terms of the harmonic tangents and gradients introduced by Teplyaev in [9]. Using the spectral decimation method of Fukushima and Shima [1] (see also Chapter 3 of [8]) we give infinite product formulae for the tangents at boundary points and use them to describe the one-sided tangents at junction points. These results may be seen as a Sierpinski Gasket version of the well-known formulae for the derivatives of the sine and cosine functions on an interval, though the precise analogue on $[0,1]$ is more complicated (see Equations 2.2 2.4). Calculations of this type are significant in the setting of p.c.f. self-similar fractals like SG because of the importance of recursive self-similar constructions that rely on matching properties of the harmonic tangent and gradient at junction points. The best known of these is Kigami's construction of the Green's function [3]. Our results should allow these methods to be generalized from piecewise harmonic functions to piecewise eigenfunctions. In particular, they will be used in a forthcoming paper [2] to obtain the resolvent of the Laplacian on SG by a method analogous to that used by Kigami.

The Sierpinski Gasket is the simplest non-trivial example of a fractal to which the standard theory of analysis on fractals applies. We refer to the monographs [3, 8]

Received by the editors November 14, 2007.

2000 Mathematics Subject Classification. Primary 28A80; Secondary 33E30.

The research of the first author was supported by the National Science Foundation through the Research Experiences for Undergraduates (REU) Program at Cornell University.

The research of the third author was supported in part by the National Science Foundation, Grant DMS-0652440.

(C)2008 American Mathematical Society Reverts to public domain 28 years from publication 
for detailed proofs of all results we use from this theory. Recall that $\mathrm{SG} \subset \mathbb{R}^{2}$ is the attractor of an iterated function system consisting of three maps $F_{i}(x)=\left(x+q_{i}\right) / 2$, where the points $q_{0}, q_{1}$ and $q_{2}$ are the vertices of an equilateral triangle. This means that $\mathrm{SG}=\bigcup_{i} F_{i}(\mathrm{SG})$, where the sets $F_{i}(\mathrm{SG})$ are usually referred to as 1-cells. For a length $m$ word $w=w_{1} \ldots w_{m}$ with letters $w_{j} \in\{0,1,2\}$ we define $F_{w}=F_{w_{1}} \circ \cdots \circ F_{w_{m}}$ and call $F_{w}(\mathrm{SG})$ an $m$-cell. The points $q_{i}, i=0,1,2$, are the boundary of SG; the set of boundary points is $V_{0}$, and we use $V_{m}$ to denote points of the form $F_{w}\left(q_{i}\right)$, where $w$ is a word of length $m$. These $V_{m}$ are vertices of the usual graph approximation of SG at scale $m$, in which vertices $x$ and $y$ are joined by an edge (written $x \sim_{m} y$ ) if they belong to a common $m$-cell. Clearly $V_{*}=\bigcup_{m} V_{m}$ is dense in SG.

The Laplacian $\Delta$ on SG is a renormalized limit of graph Laplacians $\Delta_{m}$ on the $m$-scale graphs:

$$
\begin{gathered}
\Delta u(x)=\frac{3}{2} \lim _{m \rightarrow \infty} 5^{m} \Delta_{m} u(x), \\
\Delta_{m} u(x)=\sum_{y \sim_{m} x}(u(y)-u(x)) \quad \text { for } x \in V_{m} \backslash V_{0},
\end{gathered}
$$

and we say $u \in \operatorname{dom}(\Delta)$ if the right side of (1.1) converges uniformly on $V_{*} \backslash V_{0}$ to a continuous function. The function is extended to $\mathrm{SG}$ by continuity and density of $V_{*}$. At a boundary point $q_{i} \in V_{0}$ there is an associated normal derivative defined (with $q_{i+3}=q_{i}$ ) by

$$
\partial_{n} u\left(q_{i}\right)=\lim _{m \rightarrow \infty}\left(\frac{5}{3}\right)^{m}\left(2 u\left(q_{i}\right)-u\left(F_{i}^{m}\left(q_{i+1}\right)\right)-u\left(F_{i}^{m}\left(q_{i+2}\right)\right)\right) .
$$

A harmonic function $h$ is one for which $\Delta h=0$, and for any assignment of values on $V_{0}$ there is a unique harmonic function with these boundary values. For this reason we identify the harmonic functions with the space of functions on $V_{0}$. The harmonic functions are also graph harmonic, so it is elementary to compute the values of $h$ on $V_{1}$ from those on $V_{0}$, and recursively to obtain the values on $V_{m}$ for any $m$. It will be useful to formalize this by defining the harmonic extension matrices $A_{i}$, which map the values of $h$ on $V_{0}$ to those on $F_{i}\left(V_{0}\right)$, by

$$
\left(\begin{array}{l}
h \circ F_{i}\left(q_{0}\right) \\
h \circ F_{i}\left(q_{1}\right) \\
h \circ F_{i}\left(q_{2}\right)
\end{array}\right)=A_{i}\left(\begin{array}{l}
h\left(q_{0}\right) \\
h\left(q_{1}\right) \\
h\left(q_{2}\right)
\end{array}\right)
$$

and more generally $\left(h \circ F_{w}\left(q_{0}\right), h \circ F_{w}\left(q_{1}\right), h \circ F_{w}\left(q_{2}\right)\right)^{T}=A_{w}\left(h\left(q_{0}\right), h\left(q_{1}\right), h\left(q_{2}\right)\right)^{T}$, where $A_{w}=A_{w_{m}} A_{w_{m-1}} \cdots A_{w_{1}}$. We usually write this in the compact form

$$
\left.h\right|_{F_{w} V_{0}}=\left.A_{w} h\right|_{V_{0}} .
$$

The matrices are

$$
A_{0}=\frac{1}{5}\left(\begin{array}{lll}
5 & 0 & 0 \\
2 & 2 & 1 \\
2 & 1 & 2
\end{array}\right), \quad A_{1}=\frac{1}{5}\left(\begin{array}{lll}
2 & 2 & 1 \\
0 & 5 & 0 \\
1 & 2 & 2
\end{array}\right), \quad A_{2}=\frac{1}{5}\left(\begin{array}{lll}
2 & 1 & 2 \\
1 & 2 & 2 \\
0 & 0 & 5
\end{array}\right) .
$$

The structure of the eigenfunctions of the Laplacian is similar to that of the harmonic functions. Specifically, it is true on SG that if $m$ is sufficiently large, then the restriction of a function $u$ satisfying $-\Delta u=\lambda u$ from SG to $V_{m}$ gives an 
eigenfunction of the graph Laplacian $-\Delta_{m} u=\lambda_{m} u$, with

$$
\begin{gathered}
\lambda_{m}\left(5-\lambda_{m}\right)=\lambda_{m-1}, \\
\lambda=\frac{3}{2} \lim _{m \rightarrow \infty} 5^{m} \lambda_{m} .
\end{gathered}
$$

Note that (1.3) implies that $\lambda_{m}$ is one of $\frac{1}{2}\left(5 \pm \sqrt{25-4 \lambda_{m-1}}\right)$, but the positive root is permitted to occur only for finitely many values of $m$ in order that the limit in (1.4) exists. This spectral decimation property was first recognized by Fukushima and Shima 1]. It is not true on all fractals, but on those where it is valid, it gives both a method for computing the spectrum and a recursion for the eigenfunctions [4]. Let us define

$$
\begin{aligned}
& A_{0}(\lambda)=\frac{1}{(5-\lambda)(2-\lambda)}\left(\begin{array}{ccc}
(5-\lambda)(2-\lambda) & 0 & 0 \\
(4-\lambda) & (4-\lambda) & 2 \\
(4-\lambda) & 2 & (4-\lambda)
\end{array}\right) \\
& A_{1}(\lambda)=\frac{1}{(5-\lambda)(2-\lambda)}\left(\begin{array}{ccc}
(4-\lambda) & (4-\lambda) & 2 \\
0 & (5-\lambda)(2-\lambda) & 0 \\
2 & (4-\lambda) & (4-\lambda)
\end{array}\right) \\
& A_{2}(\lambda)=\frac{1}{(5-\lambda)(2-\lambda)}\left(\begin{array}{ccc}
(4-\lambda) & 2 & (4-\lambda) \\
2 & (4-\lambda) & (4-\lambda) \\
0 & 0 & (5-\lambda)(2-\lambda)
\end{array}\right)
\end{aligned}
$$

provided $\lambda \neq 2,5$. The essence of the spectral decimation method on SG may then be summarized in the following theorem, which we have taken from Sections 3.2 and 3.3 of $[8$.

Theorem 1.1 (Spectral Decimation Method). If $(\Delta+\lambda) u=0$, then there is a sequence $\left\{\lambda_{m}\right\}_{m \geq m_{0}}$ satisfying (1.3) and (1.4), and such that $\lambda_{m} \neq 2,5,6$ for $m>m_{0}$, with the property that $\left.\left(\Delta_{m}+\lambda_{m}\right) u\right|_{V_{m}}=0$ for all $m \geq m_{0}$. The values of $u$ on $V_{m}$ for $m>m_{0}$ can be constructed recursively using the matrices from (1.5), (1.6), and (1.7) as follows. If $w=w_{1} w_{2} \ldots w_{m}$, then

$$
\left.u\right|_{F_{w}\left(V_{0}\right)}=\left.A_{w_{m}}\left(\lambda_{m}\right) A_{w_{m-1}}\left(\lambda_{m-1}\right) \cdots A_{w_{m_{0}+1}}\left(\lambda_{m_{0}+1}\right) u\right|_{F_{w_{1} \ldots w_{m_{0}}}\left(V_{0}\right)},
$$

and we call this the spectral decimation relation.

If $\lambda$ is not a Dirichlet eigenvalue, then we may assume $m_{0}=0$, at which point the condition $\left.\left(\Delta_{m_{0}}+\lambda_{m_{0}}\right) u\right|_{V_{m_{0}}}=0$ is taken to be vacuous. The corresponding eigenspace is 3-dimensional and parametrized by the values of $u$ on $V_{0}$.

If $\lambda$ is Dirichlet, several possibilities occur. We indicate the initial configurations, all of which may then be continued by the spectral decimation formula. Spanning sets for the configurations when $m_{0}=1$ are shown in Figure 1. The one on the left has $\lambda_{1}=2$ while those on the right have $\lambda_{1}=5$. If $m_{0} \geq 2$, then $\lambda_{m_{0}}=5$ or $\lambda_{m_{0}}=6$, and in the latter case $\lambda_{m_{0}+1}=3$. Those with $\lambda_{m_{0}}=5$ are formed from scaled and rotated copies of the functions on the right in Figure 1, arranged so that their normal derivatives cancel. A basis of chains for $m_{0}=2$ is shown in Figure 2: those for general $m$ are naturally indexed by the loops in $V_{m}$, plus two strands connecting points of $V_{0}$. In the case $\lambda_{m_{0}}=6$ the eigenfunctions are indexed by points in $V_{m_{0}-1} \backslash V_{0}$; a basis is obtained by scaling and rotating two copies of the function on the left in Figure 3 and gluing them at the chosen point, as shown on the right in Figure 3 for the case $m_{0}=2$ and a point in $V_{1}$. 

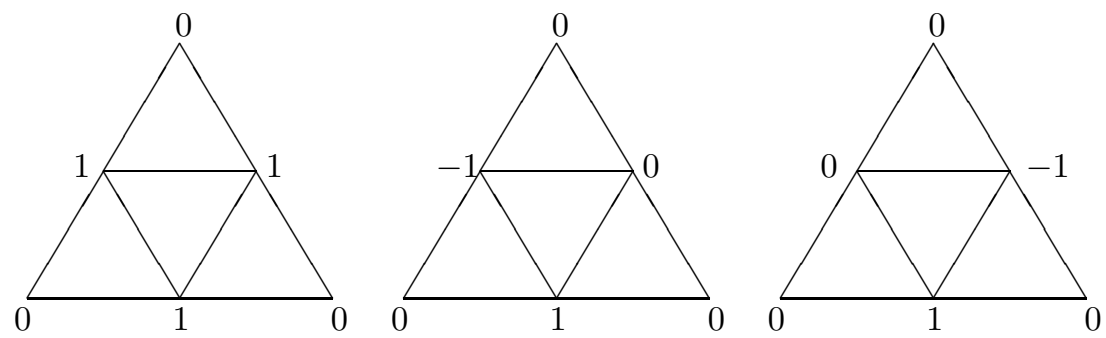

FiguRE 1. Dirichlet eigenfunctions with $m_{0}=1$
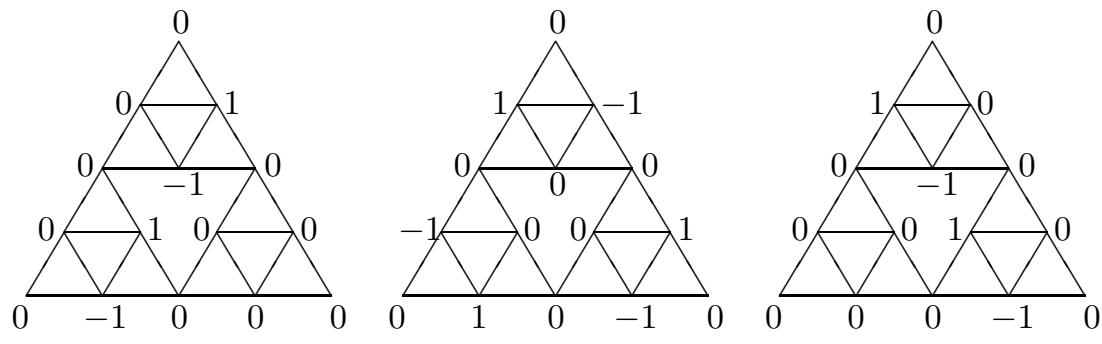

Figure 2. A basis of chains for $m_{0}=2$ and $\lambda_{m_{0}}=5$
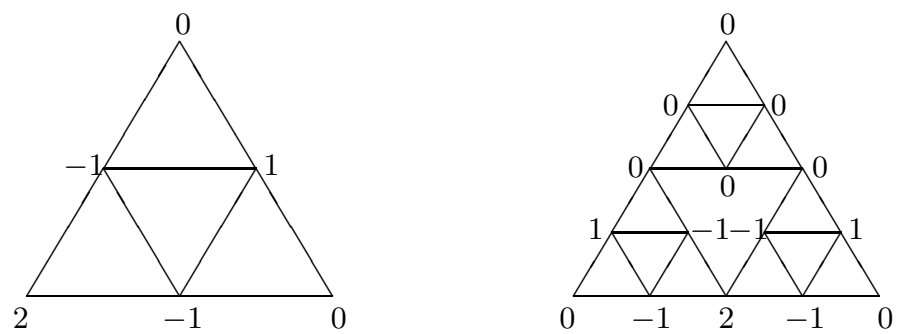

FigURE 3. Eigenfunction construction in the case $\lambda_{m_{0}}=6$

\section{TANGENTS AND GRADIENTS OF EIGENFUNCTIONS}

In 9], Teplyaev introduced the notion of a harmonic gradient and harmonic tangent for functions on the Sierpinski Gasket. He also proved that functions in the domain of the Laplacian have harmonic gradients at all points in a set of full measure and that functions with Hölder continuous Laplacian have harmonic gradients at all points. Eigenfunctions of the Laplacian fall into the latter category because of the well-known fact that continuity of $\Delta^{2} u$ implies Hölder continuity of $\Delta u([3]$, Lemma 2.2.5).

Definition 2.1. Let $w=w_{1} w_{2} \ldots$ be an infinite word, and $[w]_{m}=w_{1} w_{2} \ldots w_{m}$ be the length $m$ truncation of $w$. If $u$ is a function on SG, we let $H_{[w]_{m}} u$ be the harmonic function on $\mathrm{SG}$ which coincides with $u$ on $F_{[w]_{m}}\left(V_{0}\right)$, so

$$
H_{[w]_{m}} u=\left.A_{[w]_{m}}^{-1} u\right|_{F_{[w]_{m}}\left(V_{0}\right)}=\left.A_{w_{1}}^{-1} \cdots A_{w_{m}}^{-1} u\right|_{F_{[w]_{m}}\left(V_{0}\right)} .
$$

Define the harmonic tangent $T_{w} u$ of $u$ at $w$ to be $\lim _{m \rightarrow \infty} H_{[w]_{m}} u$ if the limit exists. It should be remarked that there can be two words $w$ and $w^{\prime}$ such that 
$F_{w}(\mathrm{SG})=F_{w^{\prime}}(\mathrm{SG})$. We will nonetheless treat the tangents $T_{w}$ and $T_{w^{\prime}}$ separately, as they are rarely equal.

The harmonic gradient $\operatorname{Grad}_{w} u$ is defined in the same way, but using the space of harmonic functions with zero mean value and the projection of the action of the matrices $A_{i}$ to this subspace. It is evident that if $u$ is continuous, then the gradient exists whenever the tangent exists, and conversely. See [7] for details.

If we consider the interval $[0,1]$ rather than the Sierpinski Gasket, then it is clear that the harmonic tangent at $x_{0}$ is the vector $(L(0), L(1))^{T}$ characterizing the unique linear function $L(x)$ having the properties $L\left(x_{0}\right)=f\left(x_{0}\right)$ and $L^{\prime}\left(x_{0}\right)=$ $f^{\prime}\left(x_{0}\right)$. For an eigenvalue $\lambda \in \mathbb{R}$ which is not equal to $\pi^{2} k^{2}$ for any $k \in \mathbb{N}$, it may readily be verified that the harmonic tangent is given by

$$
M\left(x_{0}\right)\left(\begin{array}{l}
f(0) \\
f(1)
\end{array}\right)
$$

where

$$
M\left(x_{0}\right)=\frac{1}{\sin \sqrt{\lambda}}\left(\begin{array}{ll}
a+b & a-b \\
a-b & a+b
\end{array}\right)
$$

and

$$
a=\sin \left(\left(1-x_{0}\right) \sqrt{\lambda}\right), \quad b=\sqrt{\lambda} x_{0} \cos \left(\left(1-x_{0}\right) \sqrt{\lambda}\right) .
$$

We cannot obtain as explicit a description of the harmonic tangent on SG; however, we produce formulae that permit its computation at any point of $V_{*}$. The key observation is that when $u$ is an eigenfunction, the computation of the tangent has a particularly elegant structure. Recall from Theorem 1.1 that the values of $u$ on $F_{[w]_{m}}\left(V_{0}\right)$ may be computed using the spectral decimation method, meaning that starting from a scale $m_{0}$ they can be obtained as in (1.8). Combining this with (2.1) we see that

$$
\begin{aligned}
T_{w} u= & \left.\lim _{m \rightarrow \infty} A_{w_{1}}^{-1} \cdots A_{w_{m}}^{-1} \cdot A_{w_{m}}\left(\lambda_{m}\right) \cdots A_{w_{m_{0}+1}}\left(\lambda_{m_{0}+1}\right) u\right|_{F_{w_{1} \ldots w_{m_{0}}}\left(V_{0}\right)} \\
= & \left(A_{w_{1}}^{-1} \cdots A_{w_{m_{0}}}^{-1}\right) . \\
& \left.\cdot\left(\lim _{m \rightarrow \infty} A_{w_{m_{0}+1}}^{-1} \cdots A_{w_{m}}^{-1} \cdot A_{w_{m}}\left(\lambda_{m}\right) \cdots A_{w_{m_{0}+1}}\left(\lambda_{m_{0}+1}\right)\right) u\right|_{F_{w_{1} \ldots w_{m}}\left(V_{0}\right)}
\end{aligned}
$$

in which we know the limit exists by Theorem 3 of [9]. A special case occurs when $F_{w}(\mathrm{SG})$ is a point in $V_{*}$, because in this case all but finitely many letters in the word $w$ are equal to a single letter $i$. By taking $m_{0}$ to be sufficiently large we see that it is useful to understand the limit

$$
\lim _{k \rightarrow \infty} A_{i}^{-k} \cdot A_{i}\left(\lambda_{m_{0}+k}\right) \cdots A_{i}\left(\lambda_{m_{0}+1}\right)
$$

and it is evident from the symmetry of the matrices $A_{i}$ and $A_{i}(\lambda)$ that it suffices to deal with the case $i=0$. 
Theorem 2.2. Let $\alpha=(0,1,1)^{T}, \beta=(0,1,-1)^{T}, \gamma_{m}=\left(4,4-\lambda_{m}, 4-\lambda_{m}\right)^{T}$. If neither of the values 2 or 5 occurs in the sequence $\left\{\lambda_{m}\right\}_{m>m_{0}}$, then

$$
\begin{gathered}
\lim _{k \rightarrow \infty} A_{0}^{-k} \cdot A_{0}\left(\lambda_{m_{0}+k}\right) \cdots A_{0}\left(\lambda_{m_{0}+1}\right) \alpha=\frac{4 \lambda}{3 \cdot 5^{m_{0}} \lambda_{m_{0}}\left(2-\lambda_{m_{0}+1}\right)} \prod_{j=2}^{\infty}\left(1-\frac{\lambda_{m_{0}+j}}{3}\right) \alpha, \\
\lim _{k \rightarrow \infty} A_{0}^{-k} \cdot A_{0}\left(\lambda_{m_{0}+k}\right) \cdots A_{0}\left(\lambda_{m_{0}+1}\right) \beta=\frac{2 \lambda}{3 \cdot 5^{m_{0}} \lambda_{m_{0}}} \beta, \\
\lim _{k \rightarrow \infty} A_{0}^{-k} \cdot A_{0}\left(\lambda_{m_{0}+k}\right) \cdots A_{0}\left(\lambda_{m_{0}+1}\right) \gamma_{m_{0}}=(4,4,4)^{T} .
\end{gathered}
$$

Proof. Observe that $\alpha$ and $\beta$ are eigenvectors of $A_{0}(\lambda)$, with the eigenvalues $(6-\lambda)(2-\lambda)^{-1}(5-\lambda)^{-1}$ and $(5-\lambda)^{-1}$ respectively, provided $\lambda \neq 2,5$. As $A_{0}=A_{0}(0)$ is a special case, we compute immediately that

$$
\lim _{k \rightarrow \infty} A_{0}^{-k} \cdot A_{0}\left(\lambda_{m_{0}+k}\right) \cdots A_{0}\left(\lambda_{m_{0}+1}\right) \beta=\lim _{k \rightarrow \infty} 5^{k} \prod_{j=1}^{k}\left(5-\lambda_{m_{0}+j}\right)^{-1} \beta .
$$

However, induction on (1.3) implies that $\lambda_{m_{0}}=\lambda_{m_{0}+k} \prod_{j=1}^{k}\left(5-\lambda_{m}\right)$ and therefore

$$
\lim _{k \rightarrow \infty} A_{0}^{-k} \cdot A_{0}\left(\lambda_{m_{0}+k}\right) \cdots A_{0}\left(\lambda_{m_{0}+1}\right) \beta=\lim _{k \rightarrow \infty} 5^{k} \lambda_{m_{0}+k} \lambda_{m_{0}}^{-1} \beta=\frac{2 \lambda}{3 \cdot 5^{m_{0}} \lambda_{m_{0}}} \beta .
$$

The corresponding computation for $\alpha$ can be simplified by observing from (1.3) that $\left(6-\lambda_{m}\right)=\left(3-\lambda_{m+1}\right)\left(2-\lambda_{m+1}\right)$, whereupon

$$
\begin{aligned}
\lim _{k \rightarrow \infty} & A_{0}^{-k} \cdot A_{0}\left(\lambda_{m_{0}+k}\right) \cdots A_{0}\left(\lambda_{m_{0}+1}\right) \alpha \\
& =\lim _{k \rightarrow \infty}\left(\frac{5}{3}\right)^{k} \prod_{j=1}^{k} \frac{\left(6-\lambda_{m_{0}+j}\right)}{\left(5-\lambda_{m_{0}+j}\right)\left(2-\lambda_{m_{0}+j}\right)} \alpha \\
& =\lim _{k \rightarrow \infty}\left(\frac{2-\lambda_{m_{0}+k}}{2-\lambda_{m_{0}+1}}\right)\left(5^{k} \prod_{j=1}^{k} \frac{1}{5-\lambda_{m_{0}+j}}\right)\left(3^{-k} \prod_{j=1}^{k}\left(3-\lambda_{m_{0}+j+1}\right)\right) \alpha \\
& =\left(\frac{2}{2-\lambda_{m_{0}+1}}\right)\left(\frac{2 \lambda}{3 \cdot 5^{m_{0}} \lambda_{m_{0}}}\right) \prod_{j=2}^{k+1}\left(1-\frac{\lambda_{m_{0}+j}}{3}\right) \alpha,
\end{aligned}
$$

where we used the previously computed limit for the middle factor and the fact that $\lambda_{m}=O\left(5^{-m}\right)$ as $m \rightarrow \infty$ from (1.4).

For $\gamma_{m}$ the situation is a little different. Observe that

$$
A_{0}\left(\lambda_{m+1}\right) \gamma_{m}=\left(2-\lambda_{m+1}\right)^{-1}\left(5-\lambda_{m+1}\right)^{-1}\left(\begin{array}{c}
4\left(2-\lambda_{m+1}\right)\left(5-\lambda_{m+1}\right) \\
4\left(4-\lambda_{m+1}\right)+\left(4-\lambda_{m}\right)\left(6-\lambda_{m+1}\right) \\
4\left(4-\lambda_{m+1}\right)+\left(4-\lambda_{m}\right)\left(6-\lambda_{m+1}\right)
\end{array}\right) .
$$

However, we can perform the following simplification from (1.3):

$$
\begin{aligned}
4(4- & \left.\lambda_{m+1}\right)+\left(4-\lambda_{m}\right)\left(6-\lambda_{m+1}\right) \\
& =4\left(4-\lambda_{m+1}\right)+\left(4-5 \lambda_{m+1}+\lambda_{m+1}^{2}\right)\left(6-\lambda_{m+1}\right) \\
& =4\left(4-\lambda_{m+1}\right)+\left(4-\lambda_{m+1}\right)\left(1-\lambda_{m+1}\right)\left(6-\lambda_{m+1}\right) \\
& =\left(4-\lambda_{m+1}\right)\left(10-7 \lambda_{m+1}+\lambda_{m+1}^{2}\right) \\
& =\left(4-\lambda_{m+1}\right)\left(5-\lambda_{m+1}\right)\left(2-\lambda_{m+1}\right) .
\end{aligned}
$$


Inserting this into the previous computation shows that $A_{0}\left(\lambda_{m+1}\right) \gamma_{m}=\gamma_{m+1}$ provided $\lambda_{m} \neq 2,5$, and therefore $A_{0}\left(\lambda_{m_{0}+k}\right) \cdots A_{0}\left(\lambda_{m_{0}+1}\right) \gamma_{m_{0}}=\gamma_{m_{0}+k}$. To proceed we must apply $A_{0}^{-k}$ to $\gamma_{m_{0}+k}$, which is most easily done by writing it in terms of eigenvectors as $\gamma_{m_{0}+k}=(4,4,4)^{T}-\lambda_{m_{0}+k} \alpha$. The result is

$$
\begin{aligned}
\lim _{k \rightarrow \infty} A_{0}^{-k} \cdot A_{0}\left(\lambda_{m_{0}+k}\right) \cdots A_{0}\left(\lambda_{m_{0}+1}\right) \gamma_{m_{0}} & =\lim _{k \rightarrow \infty} A_{0}^{-k}\left((4,4,4)^{T}-\lambda_{m_{0}+k} \alpha\right) \\
& =\lim _{k \rightarrow \infty}\left((4,4,4)^{T}-\left(\frac{5}{3}\right)^{k} \lambda_{m_{0}+k}\right) \\
& =(4,4,4)^{T}
\end{aligned}
$$

because $\lambda_{m}=O\left(5^{-m}\right)$ as $m \rightarrow \infty$.

Theorem 2.3. Suppose that $w$ is a word of the form $w=[w]_{k_{0}} 000 \cdots$, that $(\Delta+\lambda) u=0$ and that $m_{0}$ is chosen large enough that the spectral decimation formula holds with $\lambda_{m} \neq 2,5,6$ when $m>m_{0}$. If $k=\max \left\{k_{0}, m_{0}\right\}$, then

$$
T_{w} u=\left.\left(A_{w_{1}}^{-1} \cdots A_{w_{k}}^{-1}\right) M_{0}(\lambda, k) A_{w_{k}}\left(\lambda_{k}\right) \cdots A_{w_{m_{0}+1}}\left(\lambda_{m_{0}+1}\right) u\right|_{F_{w_{1} \ldots w_{m_{0}}}\left(V_{0}\right)}
$$

where

$$
M_{0}(\lambda, k)=\left(\begin{array}{ccc}
1 & 0 & 0 \\
1-\frac{\left(4-\lambda_{k}\right) \lambda \tau_{k}(\lambda)}{3 \cdot 5^{k} \lambda_{k}} & \frac{\lambda\left(2 \tau_{k}(\lambda)+1\right)}{3 \cdot 5^{k} \lambda_{k}} & \frac{\lambda\left(2 \tau_{k}(\lambda)-1\right)}{3 \cdot 5^{5} \lambda_{k}} \\
1-\frac{\left(4-\lambda_{k}\right) \lambda \lambda_{k}(\lambda)}{3 \cdot 5^{k} \lambda_{k}} & \frac{\lambda\left(2 \tau_{k}(\lambda)-1\right)}{3 \cdot 5^{k} \lambda_{k}} & \frac{\lambda\left(2 \tau_{k}(\lambda)+1\right)}{3 \cdot 5^{5} \lambda_{k}}
\end{array}\right)
$$

and

$$
\tau_{k}(\lambda)=\frac{1}{\left(2-\lambda_{k+1}\right)} \prod_{j=2}^{\infty}\left(1-\frac{\lambda_{k+j}}{3}\right)
$$

Proof. From (2.5) we have

$$
\begin{aligned}
T_{w} u= & \left.\left(A_{w_{1}}^{-1} \cdots A_{w_{k}}^{-1}\right)\left(\lim _{m \rightarrow \infty} A_{0}^{-(m-k)} \cdot A_{0}\left(\lambda_{m}\right) \cdots A_{0}\left(\lambda_{k+1}\right)\right) u\right|_{F_{w_{1} \ldots w_{k}}\left(V_{0}\right)} \\
= & \left(A_{w_{1}}^{-1} \cdots A_{w_{k}}^{-1}\right)\left(\lim _{m \rightarrow \infty} A_{0}^{-(m-k)} \cdot A_{0}\left(\lambda_{m}\right) \cdots A_{0}\left(\lambda_{k+1}\right)\right) \\
& \left.\cdot A_{w_{k}}\left(\lambda_{k}\right) \cdots A_{w_{m_{0}+1}}\left(\lambda_{m_{0}+1}\right) u\right|_{F_{w_{1} \ldots w_{m_{0}}}\left(V_{0}\right)}
\end{aligned}
$$

because $w_{j}=0$ for $j \geq k$ and spectral decimation applies for $j \geq m_{0}$. The result is therefore equivalent to

$$
M_{0}=\lim _{m \rightarrow \infty} A_{0}^{-(m-k)} \cdot A_{0}\left(\lambda_{m}\right) \cdots A_{0}\left(\lambda_{k+1}\right),
$$

which follows from Theorem 2.2 ,

Projection onto the subspace of harmonic functions with mean zero immediately yields the harmonic gradient. 
Corollary 2.4. Under the hypotheses of Theorem 2.3, the harmonic gradient is given by

$$
\begin{aligned}
\operatorname{Grad}_{w} u= & \left.\left(A_{w_{1}}^{-1} \cdots A_{w_{k}}^{-1}\right) M_{0}(\lambda, k) A_{w_{k}}\left(\lambda_{k}\right) \cdots A_{w_{m_{0}+1}}\left(\lambda_{m_{0}+1}\right) u\right|_{F_{w_{1} \ldots w_{m_{0}}}\left(V_{0}\right)} \\
& -\left(\left(1-\frac{2\left(4-\lambda_{k}\right) \lambda \tau_{k}(\lambda)}{3^{2} 5^{k} \lambda_{k}}\right), \quad \frac{4 \lambda \tau_{k}(\lambda)}{3^{2} 5^{k} \lambda_{k}}, \frac{4 \lambda \tau_{k}(\lambda)}{3^{2} 5^{k} \lambda_{k}}\right) \\
& \left.\cdot A_{w_{k}}\left(\lambda_{k}\right) \cdots A_{w_{m_{0}+1}}\left(\lambda_{m_{0}+1}\right) u\right|_{F_{w_{1} \ldots w_{m_{0}}}\left(V_{0}\right)}\left(\begin{array}{l}
1 \\
1 \\
1
\end{array}\right) .
\end{aligned}
$$

Proof. We obtain $\operatorname{Grad}_{w} u$ from $T_{w} u$ by subtracting the mean value. Since all of the $A_{i}$ matrices preserve constants, it suffices to find the mean value of

$$
\left.M_{0}(\lambda, k) A_{w_{k}}\left(\lambda_{k}\right) \cdots A_{w_{m_{0}+1}}\left(\lambda_{m_{0}+1}\right) u\right|_{F_{w_{1} \ldots w_{m_{0}}}\left(V_{0}\right)},
$$

which is simply

$$
\left.\left(1-\frac{2\left(4-\lambda_{k}\right) \lambda \tau_{k}(\lambda)}{3^{2} 5^{k} \lambda_{k}}, \frac{4 \lambda \tau_{k}(\lambda)}{3^{2} 5^{k} \lambda_{k}}, \quad \frac{4 \lambda \tau_{k}(\lambda)}{3^{2} 5^{k} \lambda_{k}}\right) \cdot A_{w_{k}}\left(\lambda_{k}\right) \cdots A_{w_{m_{0}+1}}\left(\lambda_{m_{0}+1}\right) u\right|_{F_{w_{1} \ldots w_{m_{0}}}\left(V_{0}\right)} .
$$

Remark 2.5. The function $\tau_{k}(\lambda)$ may appear to depend on the sequence $\left\{\lambda_{j}\right\}$, but in fact this sequence is uniquely determined by $\lambda$. Indeed, there is an entire analytic function $\Psi(z)$ with the property that $\lambda_{j}=\Psi\left(5^{-j} \lambda\right)$. To see this, let $\psi(z)=z(5-z)$ and $\psi_{m}(z)=\psi^{\circ m}\left(\frac{2}{3} 5^{-m} z\right)$. The sequence $\psi_{m}(z)$ consists of entire functions with $\psi_{m}(0)=0$ and $\psi_{m}^{\prime}(0)=\frac{2}{3}$, so is normal with limit $\Psi(z)$, a power series for which may be computed recursively. It is then clear that $\Psi\left(5^{-j} \lambda\right)=\lim _{m} \Psi\left(\frac{3}{2} 5^{m-j} \lambda_{m}\right)=$ $\lim _{m} \psi_{m-j}\left(\lambda_{m}\right)=\lambda_{j}$. Moreover, we may define

$$
\Upsilon(\lambda)=\frac{1}{\left(2-\Psi\left(5^{-1} \lambda\right)\right)} \prod_{j=2}^{\infty}\left(1-\frac{\Psi\left(5^{-j} \lambda\right)}{3}\right),
$$

at which point $\tau_{k}(\lambda)=\Upsilon\left(5^{-k} \lambda\right)$. In the same way that certain functions (e.g. Bessel and Gamma functions) that frequently arise in the study of differential equations in Euclidean analysis are termed "special functions", we suggest that the common occurrence of the functions $\Psi(\lambda)$ and $\Upsilon(\lambda)$ in analyzing differential equations on SG (see for example [5, 6]) qualifies them as special functions in analysis on the Sierpinski Gasket. In terms of these functions, (2.7) has the form

$$
M_{0}(\lambda, k)=\left(\begin{array}{ccc}
1 & 0 & 0 \\
1-\frac{\lambda\left(4-\Psi\left(5^{-k} \lambda\right)\right) \Upsilon\left(5^{-k} \lambda\right)}{3 \cdot 5^{k} \Psi\left(5^{-k} \lambda\right)} & \frac{\lambda\left(2 \Upsilon\left(5^{-k} \lambda\right)+1\right)}{3 \cdot 5^{k} \Psi\left(5^{-k} \lambda\right)} & \frac{\lambda\left(2 \Upsilon\left(5^{-k} \lambda\right)-1\right)}{3 \cdot 5^{k} \Psi\left(5^{-k} \lambda\right)} \\
1-\frac{\lambda\left(4-\Psi\left(5^{-k} \lambda\right)\right) \Upsilon\left(5^{-k} \lambda\right)}{3 \cdot 5^{k} \Psi\left(5^{-k} \lambda\right)} & \frac{\lambda\left(2 \Upsilon\left(5^{-k} \lambda\right)-1\right)}{3 \cdot 5^{k} \Psi\left(5^{-k} \lambda\right)} & \frac{\lambda\left(2 \Upsilon\left(5^{-k} \lambda\right)+1\right)}{3 \cdot 5^{k} \Psi\left(5^{-k} \lambda\right)}
\end{array}\right) .
$$

As a particular consequence we may compute the normal derivatives of the eigenfunctions at points of $V_{0}$, because they are the same as the normal derivatives of the tangent functions. This is an important step in giving an explicit construction of the resolvent of the Laplacian on the Sierpinski Gasket, which will be the subject of a later paper 2 . 
Corollary 2.6. If $(\Delta+\lambda) u=0$ and the spectral decimation formula holds with $\lambda_{m} \neq 2,5,6$ for $m>0$, then the normal derivative of $u$ at $q_{0}$ is

$$
\partial_{n} u\left(q_{0}\right)=\left(\left(4-\lambda_{0}\right) u\left(q_{0}\right)-2 u\left(q_{1}\right)-2 u\left(q_{2}\right)\right) \frac{2 \lambda \Upsilon(\lambda)}{3 \lambda_{0}} .
$$

It is necessary to exclude the values 2,5 and 6 from the sequence $\left\{\lambda_{m}\right\}$ in Theorems 2.2 and 2.3 and Corollary 2.4 because they occur precisely in the Dirichlet case, where the boundary data vanishes and cannot be used to determine the tangent or gradient. Nonetheless, Theorem 2.3 may be applied to find tangents to Dirichlet eigenfunctions in a simple fashion, and Corollary 2.4 may be used in the same manner to obtain the gradients. The reason is that the description of the Dirichlet eigenfunctions given in Theorem 1.1] ensures that we need only compute the harmonic tangents and gradients of the functions in Figure 1 and the left of Figure 3. All harmonic tangents and gradients of Dirichlet eigenfunctions are then obtained from these by scaling and taking suitable linear combinations.

For the basic element used to construct the 6 -series (Figure 3) we can directly apply Theorem 2.3 or Corollary 2.4 with $\lambda_{1}=6$ and $\lambda_{2}=3$. For example, if the top vertex in Figure 3 is $q_{0}$ and $w=0 \cdots$ we have

$$
T_{w} u=\frac{\lambda}{9}\left(\begin{array}{c}
0 \\
1 \\
-1
\end{array}\right) .
$$

To calculate the harmonic tangents or gradients of the basic element of the 2-series (on the left in Figure 1) we apply Theorem 2.3 (respectively Corollary 2.4) to the function shown at the left in Figure 4, starting the spectral decimation at each of the values $\lambda_{1}=\frac{5 \pm \sqrt{17}}{2}$. For the 5 -series there are two basic elements (shown at the right in Figure 1), and we proceed by calculating for the initial configurations shown in the center and on the right of Figure 4, starting with $\lambda_{1}=\frac{5 \pm \sqrt{5}}{2}$. The harmonic tangents and gradients of all 2 and 5 -series eigenfunctions then coincide with scaled and rotated copies of these pieces and their negatives, assembled in the obvious manner.
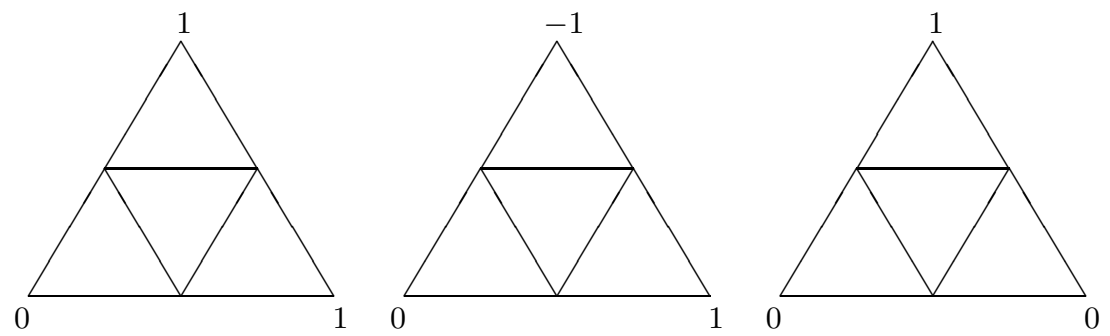

FigurE 4. Computing harmonic tangents of 2 and 5-series eigenfunctions

\section{REFERENCES}

[1] M. Fukushima and T. Shima, On a spectral analysis for the Sierpiński gasket, Potential Anal. 1 (1992), no. 1, 1-35. MR 1245223(95b:31009)

[2] Marius Ionescu, Erin P. J. Pearse, Luke G. Rogers, Hou-Jun Ruan, and Robert S. Strichartz, The resolvent of the Laplacian on p.c.f. self-similar fractals, in preparation. 
[3] Jun Kigami, Analysis on fractals, Cambridge Tracts in Mathematics, vol. 143, Cambridge University Press, Cambridge, 2001. MR1840042 (2002c:28015)

[4] Leonid Malozemov and Alexander Teplyaev, Self-similarity, operators and dynamics, Math. Phys. Anal. Geom. 6 (2003), no. 3, 201-218. MR1997913 (2004d:47012)

[5] Jonathan Needleman, Robert S. Strichartz, Alexander Teplyaev, and Po-Lam Yung, Calculus on the Sierpinski gasket. I. Polynomials, exponentials and power series, J. Funct. Anal. 215 (2004), no. 2, 290-340. MR2150975

[6] Kasso A. Okoudjou, Luke G. Rogers, and Robert S. Strichartz, Generalized eigenfunctions and a Borel theorem on the Sierpinski gasket, Canad. Math. Bull., to appear; available at http://www. journals.cms.math.ca/cgi-bin/vault/viewprepub/okoudjou8913.prepub.

[7] Robert S. Strichartz, Taylor approximations on Sierpinski gasket type fractals, J. Funct. Anal. 174 (2000), no. 1, 76-127. MR.1761364 (2001i:31018)

[8] - Differential equations on fractals, A tutorial, Princeton University Press, Princeton, NJ, 2006. MR.2246975 (2007f:35003)

[9] Alexander Teplyaev, Gradients on fractals, J. Funct. Anal. 174 (2000), no. 1, 128-154. MR $1761365(2001 \mathrm{~h}: 31012)$

Department of Mathematics, Cornell University, Ithaca, New York 14853-4201

E-mail address: jld69@cornell.edu

Department of Mathematics, University of Connecticut, Storrs, Connecticut 062693009

E-mail address: rogers@math.uconn.edu

Department of Mathematics, Cornell University, Ithaca, New York 14853-4201

E-mail address: str@math.cornell.edu 